Mens

Revue d'histoire intellectuelle et culturelle

mens

\title{
Andrée Lévesque. Éva Circé-Côté : libre-penseuse (1871-1949), Montréal, Éditions du remue-ménage, 2010, 478 p.
}

\section{Sophie Doucet}

Volume 12, numéro 2, printemps 2012

URI : https://id.erudit.org/iderudit/1013877ar

DOI : https://doi.org/10.7202/1013877ar

Aller au sommaire du numéro

Éditeur(s)

Centre de recherche en civilisation canadienne-française

ISSN

1492-8647 (imprimé)

1927-9299 (numérique)

Découvrir la revue

Citer ce compte rendu

Doucet, S. (2012). Compte rendu de [Andrée Lévesque. Éva Circé-Côté :

libre-penseuse (1871-1949), Montréal, Éditions du remue-ménage, 2010, 478 p.]

Mens, 12(2), 165-169. https://doi.org/10.7202/1013877ar d'utilisation que vous pouvez consulter en ligne.

https://apropos.erudit.org/fr/usagers/politique-dutilisation/ 


\section{Comptes rendus}

\section{Andrée Lévesque. Éva Circé-Côté : libre-penseuse (1871- 1949), Montréal, Éditions du remue-ménage, 2010, 478 p.}

Andrée Lévesque est l'une des grandes spécialistes de l'histoire des femmes et de la gauche au Québec. On lui doit le classique La norme et les déviantes: des femmes au Québec pendant l'entre-deux-guerres (1989) et, plus récemment, Scènes de la vie en rouge: l'époque de Jeanne Corbin, 1906-1944 (1999). Elle signe, avec cette biographie de la journaliste et bibliothécaire iconoclaste Éva Circé-Côté, un ouvrage qui apporte une pierre importante à l'histoire des femmes, à l'histoire du journalisme et à l'histoire intellectuelle du Québec.

Éva Circé-Côté est demeurée longtemps inconnue des historiens alors que plusieurs femmes journalistes et/ou militantes de sa génération (Joséphine Marchand-Dandurand, Marie Gérin-Lajoie, Robertine Barry, Henriette Dessaulles) faisaient l'objet de quelques travaux en histoire et en études littéraires. Andrée Lévesque propose deux hypothèses pour expliquer ce phénomène. Premièrement, Éva Circé-Côté a elle-même brouillé les pistes en écrivant sous une dizaine de pseudonymes dans une vingtaine de publications. Deuxièmement, elle a pu être oubliée parce qu'elle ne cadre pas avec le portrait que les historiens voulaient faire de son époque.

Andrée Lévesque admet avoir eu l'ambition, avec cette biographie, "d'ouvrir une fenêtre sur le Québec progressiste des premières décennies du $\mathrm{xx}^{\mathrm{e}}$ siècle ». Cet ouvrage est en effet plus qu'une biographie, un nouvel éclairage porté sur toute une société. Effectivement, une femme qui gagne sa vie de façon autonome par des activités de nature intellectuelle, qui exprime des opinions très progressistes sur la prostitution, l'immigration, le travail des femmes, l'homosexualité, qui fait à son mari décédé des funérailles civiles (et le fait incinérer) n’est imaginable que dans un Québec moins replié 
sur lui-même, moins axé sur les traditions et moins entièrement dominé par la religion qu'on le croyait.

L'ouvrage d'Andrée Lévesque est divisé en deux grandes parties. La première est la biographie proprement dite, basée sur un éventail de sources : écrits publiés de la journaliste et écrivaine, archives des institutions qu'elle a fréquentées et, à défaut d'archives personnelles laissées par Circé-Côté elle-même, archives de contemporains qu'elle a bien connus (Olivar Asselin, Marie Gérin-Lajoie, Charles Gill). La quasi-absence de documents personnels d'Éva Circé-Côté (qui n’a laissé à la postérité que cinq longues lettres à un ami, retrouvées par l'historienne grâce à une annonce dans les journaux) a obligé à faire la part belle aux 1760 chroniques publiées pendant une période de 40 ans.

Cette partie biographique compte cinq chapitres. Le premier nous montre l'évolution de cette fille de marchand d'habits, qui passe de couventine à jeune femme fréquentant la jeunesse bohème de l'École littéraire de Montréal (elle n'a toutefois pas, en tant que femme, le droit d'assister aux réunions), à jeune poète et chroniqueuse dans Les Débats, L'Avenir, L'Avenir du Nord. Puis, on la voit décrocher le poste de bibliothécaire et conservatrice à la nouvelle bibliothèque publique de Montréal et devenir une personnalité de la scène culturelle l'année où elle lance son recueil de poèmes Bleu, Blanc, Rouge: Poésie, Paysages, Causeries et présente sa pièce de théâtre Hindelang et De Lorimier au Théâtre national français.

Le chapitre deux, qui décrit la période - très courte - de son mariage avec le « docteur des pauvres » Pierre-Salomon Côté, qui est décédé à 33 ans, est particulièrement intéressant. Par l'entremise de son mari, Éva fréquente un groupe de francs-maçons, dont Andrée Lévesque nous fournit une description éclairante. À travers ses chroniques, on voit ses idées se radicaliser à leur contact. C'est au cours de cette période qu' elle fonde, avec son amie journaliste Gaëtane de Montreuil, un lycée laïque féminin qui ne survivra que deux ans, mais servira de catalyseur à la création d'un premier " collège classique " 
pour filles par la Congrégation Notre-Dame en 1908. Quand son mari meurt, Éva provoque un scandale qui lui vaudra d'être ostracisée pendant des années en ne respectant pas les rites religieux traditionnels.

Les chapitres trois, quatre et cinq, qui s'intéressent à la vie d'Éva Circé-Côté à partir de sa quarantaine, sont moins collés au personnage, les documents sur cette période de sa vie étant rares. Ainsi, Andrée Lévesque s'accroche aux chroniques publiées par Circé-Côté et s'intéresse à elle en tant qu'observatrice de la ville et des citoyens qui l'habitent. On voit, à travers des extraits de ses chroniques, le Montréal de son temps, celui du tramway, de l'électricité nouvelle, des cinémas, des marchés, de l'immigration, etc. On voit aussi sa carrière de femme de lettres évoluer à travers ses publications, notamment, en 1924, une biographie de Louis-Joseph Papineau intitulée Papineau : son influence sur la pensée canadienne: essai de psychologie historique. En 1932, à 61 ans, elle est congédiée de la bibliothèque. L'information sur sa vie intime et psychologique (santé fragile, états dépressifs, etc.) provient presque essentiellement des lettres d'Éva Circé à Marcel Dugas, qui auront été d'une importance capitale pour cette biographie (on aurait d'ailleurs apprécié pouvoir en lire de plus longs extraits).

La deuxième partie de cet ouvrage, basée sur les chroniques de la journaliste, est une sorte de radiographie de la pensée d'Éva CircéCôté, sur cinq thèmes : libéralisme, religion, patriotisme, féminisme et travail des femmes. Cette partie permet de situer Éva Circé-Côté sur l'échiquier des idéologies de son époque et de faire ressortir l'évolution de sa pensée et ses contradictions. Andrée Lévesque met bien sûr de l'avant les aspects progressistes qui sont dominants dans sa pensée : libéralisme, modernité, féminisme. Elle ne gomme toutefois pas la part qu'elle fait à des idées qui auront nettement moins bonne presse dans le Québec de la deuxième moitié du Xx ${ }^{e}$ siècle : accents d'eugénisme, sympathies duplessistes, absence de solidarité avec les autres femmes, que, sans complaisance, elle tient souvent responsables de leurs malheurs. 
Cet ouvrage recèle de grandes qualités. L'auteure a fait une recherche remarquable dans les sources secondaires et dans les archives, rognant dans tous les coins ce qui pouvait exister sur Éva Circé-Côté et réussissant par là à redonner aux Québécois un personnage important de leur histoire. L'auteure a une plume efficace, captivante et très élégante, surtout dans les premiers chapitres, de facture très soignée. L'ouvrage comporte aussi quelques défauts. L’on pourrait regretter l'absence d'intégration des deux grandes parties du livre. Par moments, l'auteure parvient à intégrer des éléments de la pensée de Circé-Côté dans la partie biographique et l'on se dit qu'il aurait été intéressant de le faire tout au long, ce qui aurait donné un ouvrage d'une plus grande cohérence et dont la lecture aurait été plus uniformément agréable. Nous aurions aussi aimé que l'auteure fasse davantage de parallèles entre Éva Circé-Côté et les autres intellectuelles et journalistes de sa génération. Par exemple, qu'elle situe plus précisément son féminisme par rapport à celui de Marie Gérin-Lajoie (reconnu comme étant davantage du maternalisme) ou son libéralisme en fonction de celui de Joséphine Marchand-Dandurand.

D'autre part, si l'iconographie (photos de famille, photos professionnelles, unes de journaux) est intéressante et vient enrichir l'ouvrage, nous aurions apprécié, dans le premier chapitre, la reproduction d'une carte de Montréal datant de cette époque, qui aurait permis au lecteur de suivre plus facilement les déplacements de la famille d'Éva Circé-Côté. Nous aurions aussi et surtout apprécié une conclusion plus substantielle (la conclusion proposée ne fait que cinq pages), qui aurait repris les lignes de force de la vie et de la pensée du personnage. Finalement, l'auteure n'a pas manqué de faire, tout au long de l'ouvrage, l'évaluation de ses sources. Néanmoins, nous nous demandons tout de même comment nous pouvons être sûrs que certaines des opinions qu'exprimait Éva étaient bien les siennes et n'étaient pas commandées par un rédacteur en chef.

Cela dit, l'ouvrage d'Andrée Lévesque est certainement l'une des plus importantes biographies de femmes intellectuelles au Québec. L'Éva Circé-Côté qu'elle nous présente est crédible et riche en nuances. 
Andrée Lévesque la compare quelques fois, de façon intéressante, à Émile Zola. Si les Français ont de grands romanciers réalistes pour leur restituer l'atmosphère des villes du XIx ${ }^{\mathrm{e}}$ siècle, les Québécois ont des chroniqueurs - comme Éva Circé - dont les écrits, précieux, font revivre un Montréal disparu.

Cet ouvrage ne s'adresse pas qu'aux historiens professionnels, mais saura plaire à un public plus large d'amateurs d'histoire du Québec et de biographies.

- Sophie Doucet

Département d'histoire

Université du Québec à Montréal

\section{Renée Legris. Histoire des genres dramatiques à la radio québécoise : sketch, radioroman, radiothéâtre, 1923-2008, Québec, Septentrion, 2011, 512 p.}

Les recherches sur la radio québécoise ont bénéficié d'une nouvelle impulsion au cours des dix dernières années. Deux titres témoignent plus particulièrement de ce renouveau critique : Hubert Aquin et la radio : une quête d'écriture (1954-1977) (Médiaspaul, 2004) de Renée Legris, ouvrage fouillé sur la carrière hertzienne de cet écrivain emblématique de la Révolution tranquille, et Histoire de la radio au Québec : information, éducation, culture (Fides, 2007) de Pierre Pagé, recension historique de la radiophonie québécoise. Avec Histoire des genres dramatiques à la radio québécoise : sketch, radioroman, radiothéâtre, 1923-2008, où sont synthétisées plusieurs années d'analyse, Renée Legris concrétise l'intérêt actuel pour le média radiophonique en examinant, avec méthode et minutie, les fictions qui ont fait les belles heures de la radio, des premiers essais à l'antenne de CKAC aux émissions plus récentes de Radio VilleMarie, en passant par la foisonnante production dramatique radiocanadienne.

L'étude de Legris, divisée en six parties, détaille d'abord les conditions d'émergence de la dramaturgie radiophonique québécoise, marquées par l'interdépendance des milieux théâtral et médiatique, 